\title{
Edge prior guided dictionary learning for quantitative susceptibility mapping reconstruction
}

\author{
Jiacheng Du ${ }^{1 \#}$, Yuxin Ji ${ }^{2 \#}$, Jiali Zhu ${ }^{2}$, Xiaoli Mai ${ }^{3}$, Junting Zou ${ }^{3}$, Yang Chen ${ }^{2}$, Ning Gu ${ }^{1}$ \\ ${ }^{1}$ The State Key Laboratory of Bioelectronics and Jiangsu Key Laboratory of Biomaterials and Devices, School of Biological Sciences and Medical \\ Engineering, Southeast University, Nanjing, China; ${ }^{2}$ The Laboratory of Image Science and Technology, Key Laboratory of Ministry of Education, \\ School of Computer Science and Engineering, Southeast University, Nanjing, China; ${ }^{3}$ The Radiology, Nanjing Drum Tower Hospital, Nanjing, \\ China
}

Contributions: (I) Conception and design: J Du, Y Ji; (II) Administrative support: N Gu, Y Chen; (III) Provision of study materials or patients: X Mai; (IV) Collection and assembly of data: J Du, Y Ji, J Zhu; (V) Data analysis and interpretation: J Du, Y Ji, J Zhu; (VI) Manuscript writing: All authors. (VII) Final approval of manuscript: All authors.

"These authors contributed equally to this work.

Correspondence to: Ning Gu. The State Key Laboratory of Bioelectronics and Jiangsu Key Laboratory of Biomaterials and Devices, School of Biological Sciences and Medical Engineering, Southeast University, Nanjing 210009, China. Email: guning@seu.edu.cn; Yang Chen. Laboratory of Image Science and Technology, Southeast University, Nanjing, China; School of Cyber Science and Engineering, Southeast University, Nanjing, China; Key Laboratory of Computer Network and Information, Southeast University, Nanjing, China; Centre de Recherche en Information Biomedicale Sino-Francais (LIA CRIBs), Rennes, France. Email: chenyang.list@seu.edu.cn.

Background: Compared with conventional magnetic resonance imaging methods, the quantitative magnetic susceptibility mapping (QSM) technique can quantitatively measure the magnetic susceptibility distribution of tissues, which has an important clinical application value in the investigations of brain micro-bleeds, Parkinson's, and liver iron deposition, etc. However, the quantitative susceptibility mapping algorithm is an ill-posed inverse problem due to the near-zero value in the dipole kernel, and high-quality QSM reconstruction with effective streaking artifact suppression remains a challenge. In recent years, the performance of sparse representation has been well validated in improving magnetic resonance image (MRI) reconstruction.

Methods: In this study, by incorporating feature learning into sparse representation, we propose an edge prior guided dictionary learning-based reconstruction method for the dipole inversion in quantitative susceptibility mapping reconstruction. The structure feature dictionary relies on magnitude images for susceptibility maps have similar structures with magnitude images, and this structure feature dictionary and edge prior information are used in the dipole inversion step.

Results: The performance of the proposed algorithm is assessed through in vivo human brain clinical data, leading to high-quality susceptibility maps with improved streaking artifact suppression, structural recovery, and quantitative metrics.

Conclusions: The proposed edge prior guided dictionary learning method for dipole inversion in QSM achieves improved performance in streaking artifacts suppression, structural recovery and deep gray matter reconstruction.

Keywords: Quantitative susceptibility mapping; structure feature dictionary; dipole inversion

Submitted Mar 09, 2021. Accepted for publication Jul 07, 2021.

doi: 10.21037/qims-21-243

View this article at: https://dx.doi.org/10.21037/qims-21-243 


\section{Introduction}

Magnetic susceptibility is a physical property of a material. When an external magnetic field is applied, different materials will produce different degrees of magnetization, which allows quantitative analysis of iron content, calcification, blood oxygen saturation, and contrast agents in tissues (1-5). Accurate reconstruction of tissue susceptibility map from local phase measurements is of great significance in clinical research such as brain micro-bleeds, Parkinson's, liver iron deposition, and hemorrhage (6-9).

Quantitative susceptibility mapping is a technique that solves the inversion problem of the magnetic field to the susceptibility source. The susceptibility map is extracted from the phase of the magnetic resonance imaging (MRI) sequence, such as gradient echo (10). However, due to the near-zero values in the dipole kernel, the dipole inversion is an ill-posed problem (3). Many methods have been proposed to solve this problem. The Thresholded K-space Division (TKD) method solves this ill-conditioned problem in the Fourier domain (11). TKD method tackles this by replacing the small values with a preset threshold in the dipole kernel. However, the selection of threshold value is data specific, and has a large effect on the susceptibility calculation, often resulting in obvious artifacts when an unmatched threshold is used (11). Some methods use least squares to minimize the difference between predicted and actual susceptibility maps, such as $(10,12-14)$. Studies $(15,16)$ also proposed some total generalized variation (TGV) methods. Study (17) proposed a preconditioned total field inversion (TFI) method and study (18) proposed a leastnorm direct dipole inversion method. In the Morphology Enabled Dipole Inversion (MEDI) method proposed in $(19,20)$, the edge information extracted from the magnitude images is incorporated into the prior term in QSM regularization. The Calculation Of Susceptibility through Multiple Orientation Sampling (COSMOS) (21) can provide high-quality magnetic susceptibility distribution, and the result of COSMOS is usually used as the gold standard in evaluating different QSM algorithms. However, the COSMOS method requires multiple orientation data, which is hard to be realized in the clinic. Some deep learning models are also used to solve this problem, such as QSMnet (22) and deepQSM (23). Such data-driven methods usually have a large demand for training data.

In recent years, methods based on sparse representation and feature learning have been well developed for CT reconstruction (24-27), and MRI reconstruction from highly under-sampled k-space data (28). Feature learning methods such as dictionary learning show better improvement in reconstruction quality than traditional sparse representation methods in MRI reconstruction (28). Additionally, the idea of edge prior is also applied to improve under-sampled MRI reconstruction $(29,30)$.

In this paper, by incorporating feature learning into sparse representation, an edge prior guided dictionary learning (EP-DL) based reconstruction is developed for the dipole inversion in quantitative susceptibility mapping reconstruction. With a 3D structure feature dictionary, a more effective sparse representation is realized for the dipole inversion method in the proposed approach. The performance of the proposed method is well validated using in vivo human brain clinical data.

\section{Methods}

\section{QSM theory}

In QSM, the local field $B$ is the convolution of the susceptibility map $\chi$ and the dipole kernel C. In the Fourier domain, $\chi$ could be expressed as:

$$
B(k)=C(k) \chi(k)
$$

Herein, $C(k)=1 / 3-k_{z}^{2} / k^{2}$ where $k$ denotes spatial vector in the Fourier domain. However, solving $\chi(k)$ from Eq. [1] is an ill-posed problem due to the near-zero values in $C(k)$. To tackle this problem, the TKD method simply applies a constant to replace the near-zero values in $C(k)$ to avoid streaking artifacts caused by noise amplification (20). Recent studies have shown that regularization QSM methods can be used to suppress the streaking artifacts by incorporating the edge information extracted from magnitude images, such as the MEDI method (20).

The MEDI method calculates the susceptibility map $\chi$ by solving minimization: $\lambda_{1}\|W(B-C \chi)\|_{2}^{2}+\|M G \chi\|_{1}$ where $\lambda_{1}$ is the regularization parameter, $W$ is the signal to noise ratio matrix. $B$ is the local field, $C$ is the dipole kernel, $M$ is the edge prior from magnitude images and $G$ is the gradient operator.

\section{EP-DL reconstruction}

The above MEDI method still suffers from over-smoothing and artifacts (31). To overcome this problem, an EP-DL reconstruction method is proposed in this study. The EPDL method is formulized as follows: 


$$
\begin{aligned}
& \min _{\chi} \lambda_{1}\|W(B-C \chi)\|_{2}^{2}+\lambda_{2} \sum_{i, j, k}^{I, J, K}\left\|E_{i, j, k} \chi-D \alpha_{i, j, k}\right\|_{2}^{2}+\|M G \chi\|_{1} \\
& \text { s.t. }\left\|\alpha_{i, j, k}\right\|_{0} \leq T \text { and }\left\|E_{i, j, k} \chi-D \alpha_{i, j, k}\right\|_{2}^{2} \leq \varepsilon
\end{aligned}
$$

Here, the fidelity term is evaluated in the spatial domain, $\lambda_{1}$ and $\lambda_{2}$ are regularization parameters, $E_{i, j, k}$ is an operator to extract a $3 \mathrm{D}$ block from $\chi$ located at $\{i, j, k\}, \mathrm{D}$ is the $3 \mathrm{D}$ feature dictionary built by the algorithm below, $\alpha_{i, j, k}$ is the sparse representation vector, $T$ is the sparsity level, $\varepsilon$ is the tolerance parameter, and all blocks are fully overlapped, which means that the distance in pixels between corresponding pixel locations in adjacent blocks is set to 1 .

\section{Construction of $3 D$ feature dictionary}

It is shown in (19) that the edges in the susceptibility maps often have similar feature distribution as the structures in the magnitude images of gradient recalled echo (GRE) obtained in the same acquisition. Magnitude images are used in dictionary learning. The block $f_{i, j, k}($ size: $N=m \times m \times h)$ is extracted only from ROI of magnitude images to avoid all-zero blocks. Each extracted 3D block is normalized by subtracting the DC component, divided by the maximum absolute value in the block, and added to the sample block array $F=\left\{f_{i, j, k}\right\} \in R^{N \times M_{1}} \quad\left(M_{1}\right.$ is the total block number).

After this, we estimate the dictionary $D \in R^{N \times M_{2}}$ from $F \in R^{N \times M_{1}} \quad\left(M_{2}\right.$ is the dictionary atom number). The dictionary $D$ and sparse representation vector $\alpha$ can be alternatively estimated by the following minimization process:

$$
\min \|F-D \alpha\|_{2}^{2} \text { s.t. }\|\alpha\|_{0} \leq T_{L}
$$

where $T_{L}$ is the parameter of sparsity level in dictionary learning.

Eq. [3] is solved by the methods of K-Singular Value Decomposition (K-SVD) and Orthogonal Matching Pursuit (OMP) (32). The implementation of dictionary construction is given as follows:

\section{D Dictionary Construction}

Input: the magnitude images of GRE data

Output: 3D feature dictionary $D$

Construction Steps:

1) Initialization: $M_{2}, N, T_{L}$;

2) Obtain the training sample block arrays $F$;

3) Dictionary learning: obtain $3 \mathrm{D}$ feature dictionary $D$ via K-SVD algorithm specified by [3].

\section{Implementation of the EP-DL reconstruction}

The implementation of the EP-DL method includes the following steps:

Step 1. Use the TKD method to obtain the initialized susceptibility map $\chi_{0}$. With dictionary $D$ and $\chi$ fixed, we solve the sparse coefficients $\alpha_{i, j, k}$ for each extracted block separately via Eq. [4] using the Batch-OMP algorithm (33).

$$
\min \alpha_{i, j, k}\left\|\alpha_{i, j, k}\right\|_{0} \text { s.t. }\left\|E_{i, j, k} \chi-D \alpha_{i, j, k}\right\|_{2}^{2} \leq \varepsilon
$$

Step 2. In (20), using a complex exponential function as a fidelity term, we obtain Eq. [5] from Eq. [2]. Then the susceptibility map $\chi$ can be updated by solving the minimization problem Eq. [5]:

$$
\begin{aligned}
& \min _{\chi} \lambda_{1}\|W(\exp (i C \chi)-\exp (i B))\|_{2}^{2}+ \\
& \lambda_{2} \sum_{i, j, k}^{I, J, K}\left\|E_{i, j, k} \chi-D \alpha_{i, j, k}\right\|_{2}^{2}+\|M G \chi\|_{1}
\end{aligned}
$$

Here the Eq. [5] can be expanded using Taylor expansion with respect to the first order at the $n$th solution:

$$
\begin{aligned}
& \arg \min _{\nabla \chi_{n}} \lambda_{1}\left\|W\left(\exp \left(i C \chi_{n}\right)\left(1+i C \nabla \chi_{n}\right)-\exp (i B)\right)\right\|_{2}^{2}+ \\
& \lambda_{2} \sum_{i, j, k}^{I, J, K}\left\|E_{i, j, k}\left(\chi+\nabla \chi_{n}\right)-D \alpha_{i, j, k}\right\|_{2}^{2}+\left\|M G\left(\chi+\nabla \chi_{n}\right)\right\|_{1}
\end{aligned}
$$

With the definition of $w=W \exp \left(i C \chi_{n}\right)$ and $b=W \exp (i B)$, the solution can be solved by setting the gradient of Eq. [6] to zero with respect to $\nabla \chi_{n}$ :

$$
\begin{gathered}
\left(2 \lambda_{1}(w C)^{T} w C+2 \lambda_{2} \sum_{i, j, k}^{I, J, K} E_{i, j, k}^{T} E_{i, j, k}+(M G)^{T} /\left|M G \chi_{n}\right| M G\right) \nabla \chi_{n} \\
=-\left(\begin{array}{l}
2 \lambda_{1} \operatorname{real}(i w C)^{T}(w-b)+2 \lambda_{2} \sum_{i, j, k}^{I, J, K} E_{i, j, k}^{T}\left(E_{i, j, k}^{T} \chi_{n}-D \alpha_{n}^{i, j, k}\right)+ \\
(M G)^{T} /\left|M G \chi_{n}\right| M G \chi_{n}
\end{array}\right)
\end{gathered}
$$

Then we calculate $\nabla \chi_{n}$ and update $\chi$ by Conjugate Gradient method as follows:

Conjugate Gradient method 


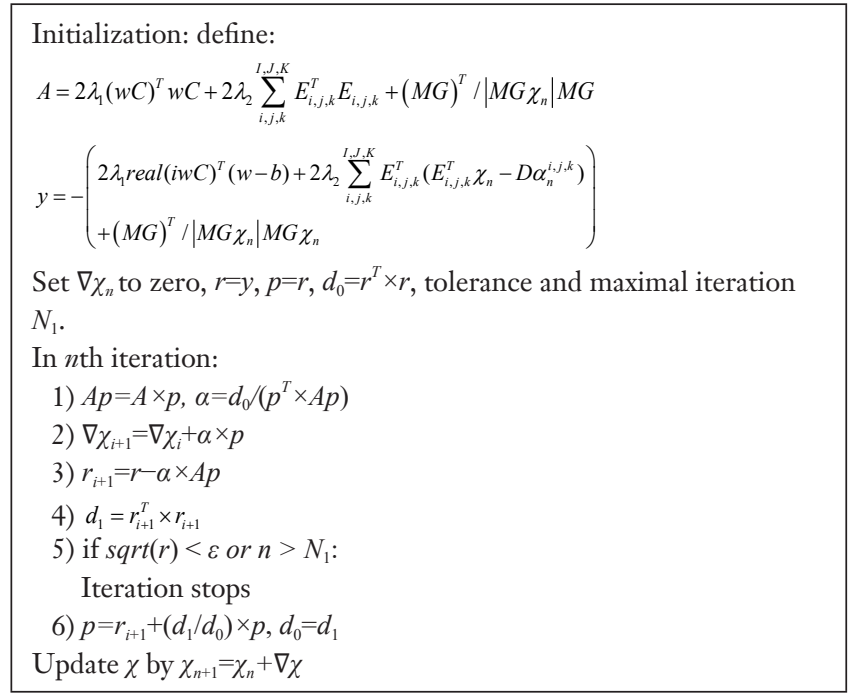

The overall EP-DL method for QSM reconstruction is outlined as follows:

EP-DL method

Input: the susceptibility mapping $\chi_{0}$ initialized via TKD algorithm;

Constructed feature dictionary $D$.

Output: reconstruction susceptibility $\chi$.

Initialization: $\lambda_{1}, \lambda_{2}$.

Iteration: repeat $n$ times

1) Feature constraint representation: updating $\alpha_{i, j, k}^{n}$ by solving the minimization problem in [4];

2) Volume updating: updating $\chi_{n}$ by solving the minimization problem in [5] via Conjugate Gradient method.

Several parameters need to be set in algorithm implementation, including the regularization parameters $\lambda_{1}, \lambda_{2}$, dictionary atom number $M_{2}$, and block size $N$. Regularization parameters $\lambda_{1}, \lambda_{2}$ are used to balance the data fidelity and two constraint terms in Eq. [6]. A reasonable block size should be used to ensure effective QSM reconstruction. Reducing block size contributes to the restoration of fine structures in the susceptibility map, but also tends to introduce high-frequency artifacts. On the other side, a large block size $N$ may lead to detail loss and increased computation cost. A large dictionary atom number $M_{2}$ may lead to increased representation accuracy and higher computation cost.

It is found in the experiment that the same parameter setting can be used for the same type of dataset. In this study, the parameters are set to give the best results in terms of RMSE and HFEN for different methods.

The implementation of EP-DL is available at https://
github.com/R1vaille/EP-DL

\section{Ethical approval}

The study was conducted in accordance with the Declaration of Helsinki (as revised in 2013). This study protocol was approved by the ethics committee of Southeast University, and written informed consent was obtained from each participant. All ethical guidelines were followed in our description of the experiments performed with human samples.

\section{Results}

\section{QSM challenge data results}

\section{Data pre-processing}

All brain masks are extracted by the BET algorithm provided by FSL Toolbox (34). For the phase of the MRI sequence, the methods of phase unwrapping and background field removal are used to preprocess the data. Unwrapping was performed using a Laplacian algorithm (35), whereas background removal was performed using the Laplacian boundary value approach (36).

\section{Experiment setting}

The public 3T human brain data in the 2016 QSM reconstruction challenge (31) and $7 \mathrm{~T}$ human brain data in the 2019 QSM reconstruction challenge (http://qsm. snu.ac.kr/?page_id=30) are used to assess the proposed EP-DL algorithm. In our method, the constructed 3D structure feature dictionary is illustrated in Figure 1. Magnitude images of GRE data are used in dictionary learning. The 3D structure feature dictionary extracts the morphological information of magnitude images. Each atom is an extracted image feature. In this experiment, to obtain the best results in terms of quantitative metrics (RMSE and HFEN), in the EP-DL method, parameter settings are shown in Table 1. MEDI Toolbox (19) is used for implementation, and the regularization parameter $\lambda_{1}$ is set to the default value 1,000 .

\section{Qualitative analysis}

Figure 2 shows typical reconstructed susceptibility maps with TKD, MEDI, EP-DL, and COSMOS methods for the 2016 QSM reconstruction challenge data in axial, sagittal, and coronal views. In Figures 2,3, fewer streaking artifacts are observed in the EP-DL method than the MEDI method 


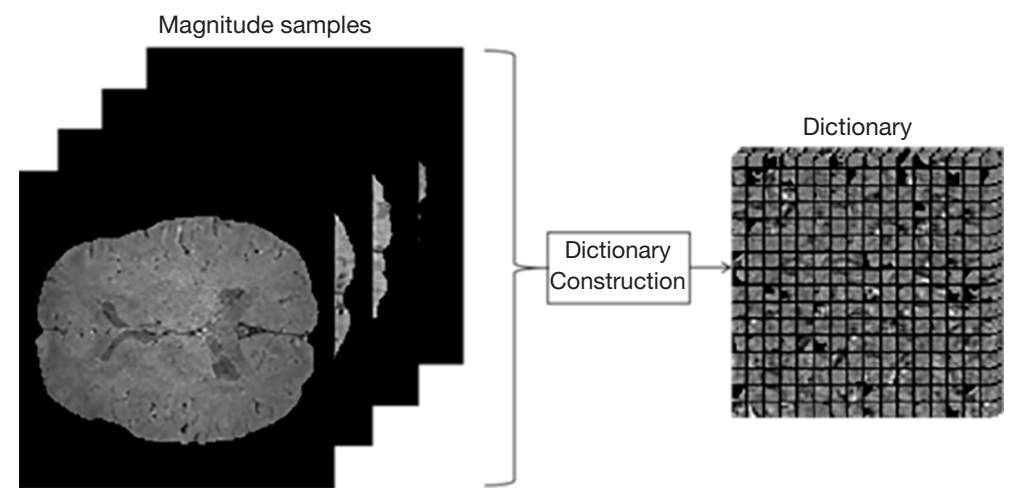

Figure 1 Construction flow chart of a 3D feature dictionary.

Table 1 Parameter settings of EP-DL method for the 2016/2019 QSM

\begin{tabular}{lcccc}
\hline Parameter & $\lambda_{1}$ & $\lambda_{2}$ & $N$ & $M_{2}$ \\
\hline 2016 & 1,200 & 60 & $4 \times 4 \times 4$ & 300 \\
2019 & 2,500 & 25 & $4 \times 4 \times 4$ & 400 \\
\hline
\end{tabular}

EP-DL, edge prior guided dictionary learning; QSM, quantitative magnetic susceptibility mapping.

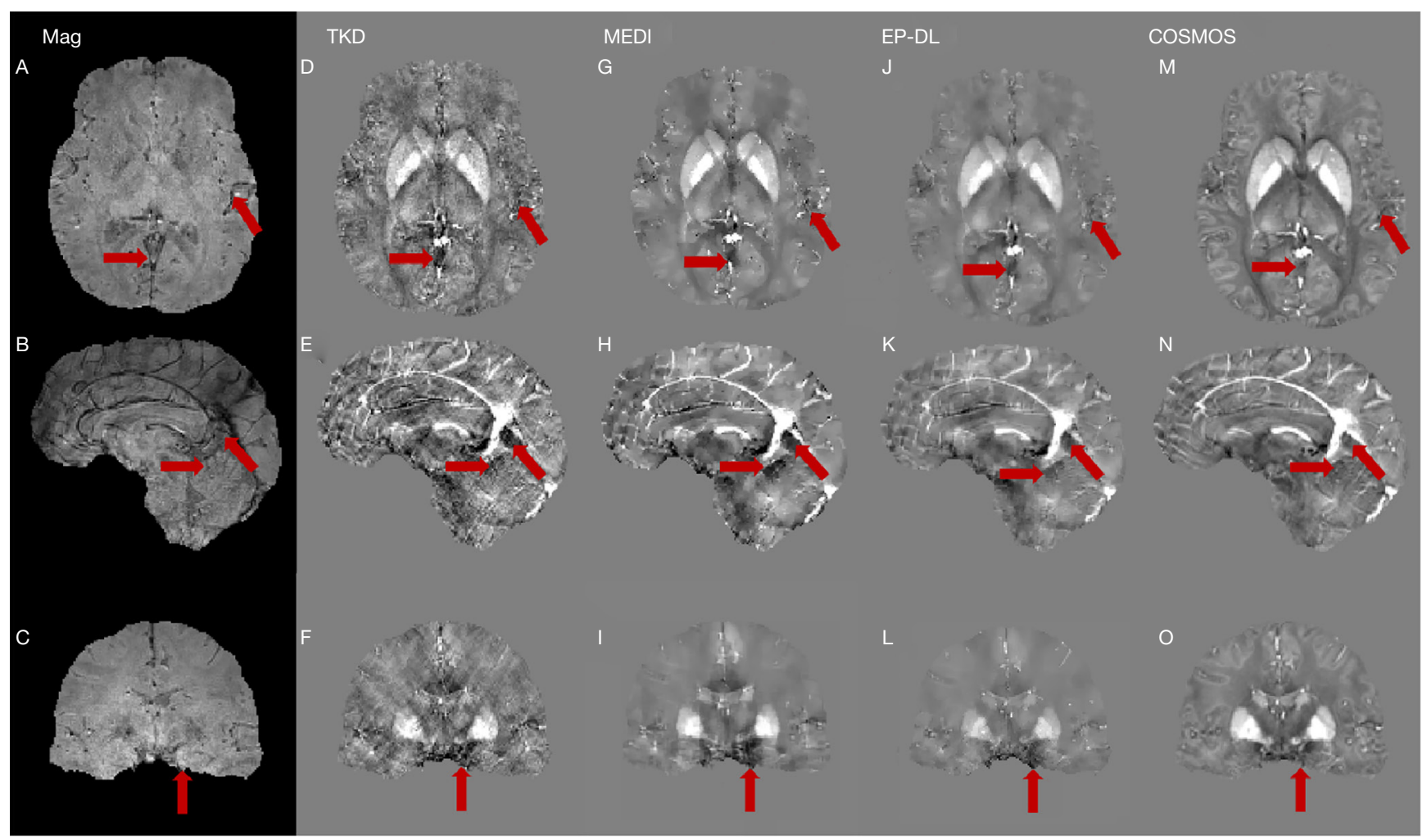

Figure 2 Magnitude images and corresponding QSM results of the 2016 QSM challenge. The first, second, and last rows are the axial, sagittal, and coronal views, from the second to the last column are the results from TKD, MEDI, EP-DL, and COSMOS methods, respectively. Red arrows are placed where the artifacts are conspicuous. The window/level is from -0.15 to 0.15 ppm. QSM, quantitative magnetic susceptibility mapping; EP-DL, edge prior guided dictionary learning; TKD, Thresholded K-space Division; MEDI, Morphology Enabled Dipole Inversion; COSMOS, Calculation Of Susceptibility through Multiple Orientation Sampling. 


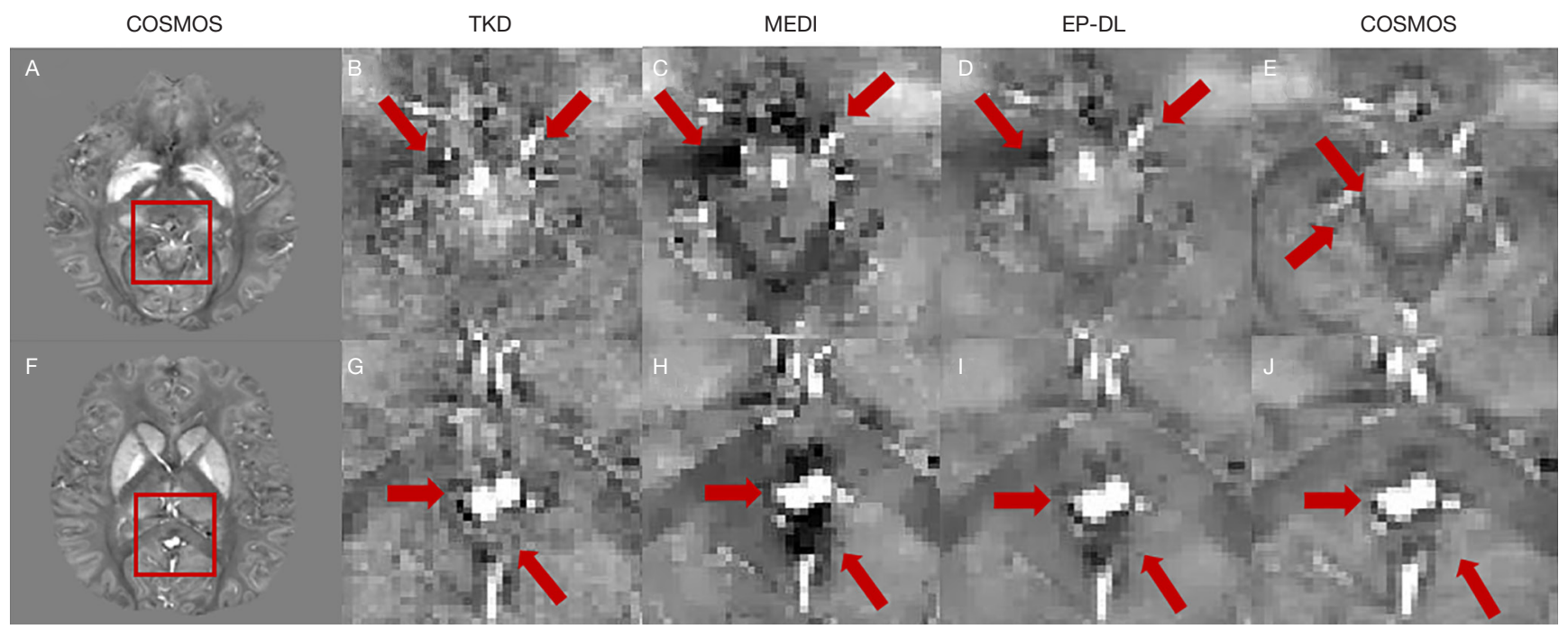

Figure 3 Axial QSM results of the 2016 QSM challenge. (B) to (E) are enlarged views of the red box in (A) and (G) to (J) are enlarged views of the red box in (B). Red arrows are placed where the artifacts are conspicuous, we can observe that artifacts in the EP-DL method are less compared with the MEDI method. The window/level is from -0.15 to $0.15 \mathrm{ppm}$. QSM, quantitative magnetic susceptibility mapping; EP-DL, edge prior guided dictionary learning; TKD, Thresholded K-space Division; MEDI, Morphology Enabled Dipole Inversion; COSMOS, Calculation Of Susceptibility through Multiple Orientation Sampling.

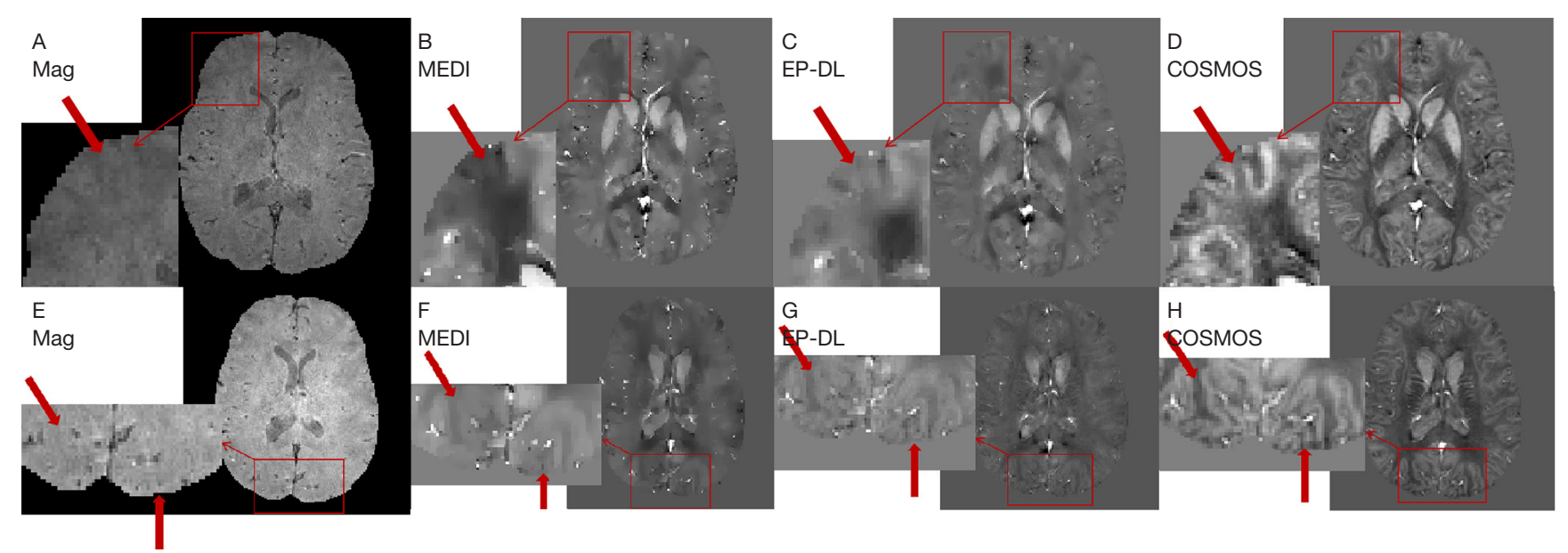

Figure 4 From the left to right column are magnitude images and corresponding QSM results of MEDI, EP-DL, and COSMOS methods, respectively. The top row (A-D) is slice 80 and the bottom row $(\mathrm{E}-\mathrm{H})$ is slice 85 , in the red rectangle, we can observe that more structural details are recovered in the EP-DL method than the MEDI method where red arrows place. QSM, quantitative magnetic susceptibility mapping; EP-DL, edge prior guided dictionary learning; MEDI, Morphology Enabled Dipole Inversion; COSMOS, Calculation Of Susceptibility through Multiple Orientation Sampling.

in contrast to the COSMOS method (see the red arrows in Figures 2,3).

Figure 4 depicts two typical slices of reconstructed susceptibility maps by MEDI, EP-DL, and COSMOS methods, the top row [Figure $4 A-4 D$ ] is the $80^{\text {th }}$ slice, and the bottom row [Figure $4 E-4 H$ ] the is $85^{\text {th }}$ slice. With the COSMOS method used as the reference, we can observe in Figure 4 that the EP-DL method preserves structural details of the cortical ribbon while the MEDI method loses some tissue information (see the red arrows). 


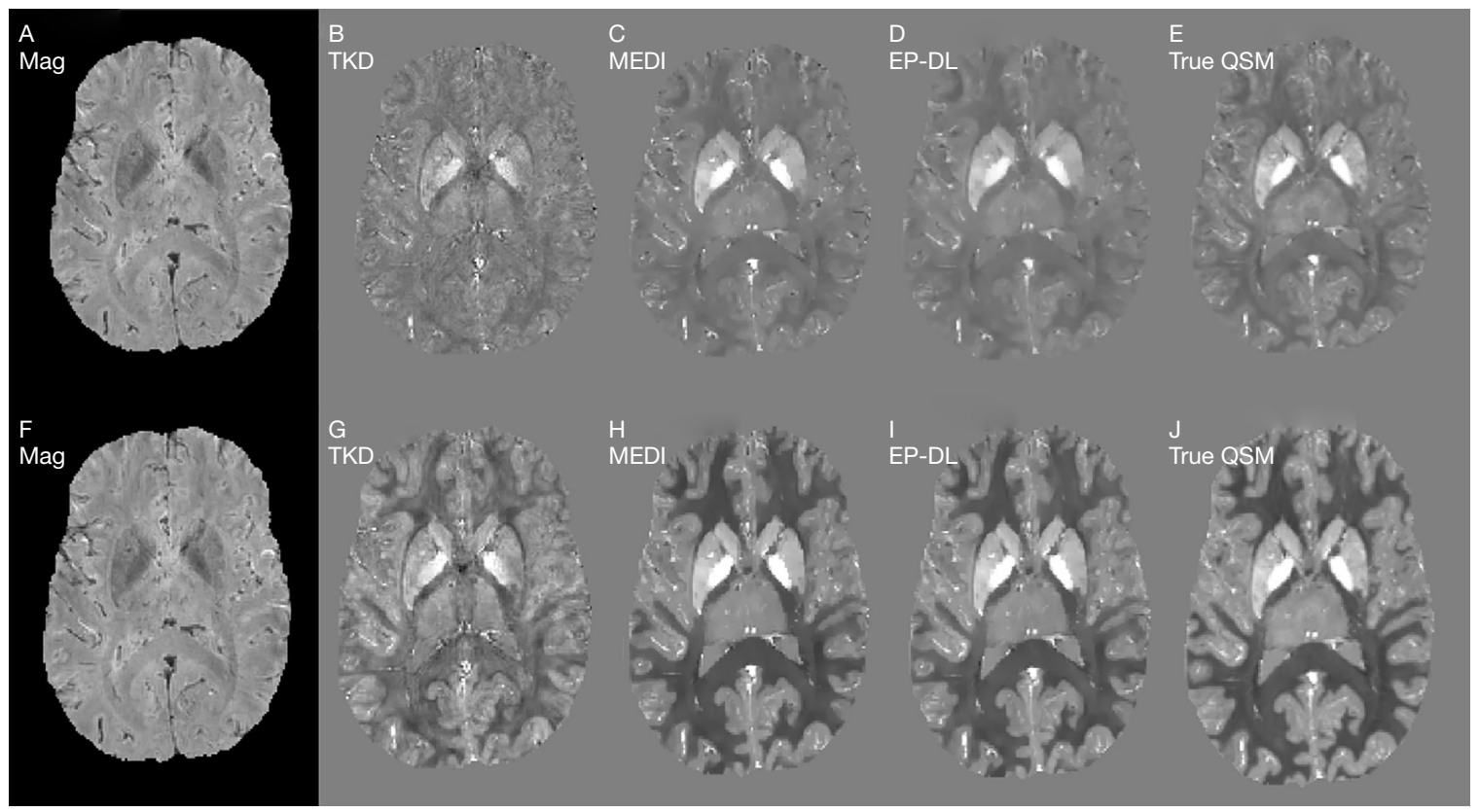

Figure 5 Magnitude images and corresponding QSM results of two data sets in the 2019 QSM challenge. From the second to the last column are the results from TKD, MEDI, EP-DL, and COSMOS methods, respectively. The window/level is from -0.15 to 0.15 ppm. QSM, quantitative magnetic susceptibility mapping; EP-DL, edge prior guided dictionary learning; TKD, Thresholded K-space Division; MEDI, Morphology Enabled Dipole Inversion; COSMOS, Calculation Of Susceptibility through Multiple Orientation Sampling.

Table 2 The RMSE and HFEN for different methods for the 2016 QSM challenge data

\begin{tabular}{lccc}
\hline Method & TKD & MEDI & EP-DL \\
\hline RMSE & 69.9 & 74.5 & 56.8 \\
HFEN & 66.7 & 64.9 & 56.1 \\
\hline
\end{tabular}

QSM, quantitative magnetic susceptibility mapping; EP-DL, edge prior guided dictionary learning; TKD, Thresholded K-space Division; MEDI, Morphology Enabled Dipole Inversion.

Figure 5 shows the reconstruction results of TKD method still have high noise, while the reconstruction results of MEDI and EP-DL method both have high reconstruction quality visually, and almost no artifacts can be observed for two data sets in the 2019 QSM reconstruction challenge.

\section{Quantitative analysis}

RMSE and HFEN with respect to the COSMOS method are calculated for quantitative performance evaluation (31). Here the RMSE is defined by Eq. [8]:

$$
\sqrt{\left\|\chi-\chi_{R}\right\|_{F}^{2} /\left\|\chi_{R}\right\|_{F}^{2}}
$$

where $\chi$ is the recovered quantitative susceptibility map,
Table 3 The RMSE and HFEN for different methods for two data sets in the 2019 QSM challenge

\begin{tabular}{llccc}
\hline Method & & TKD & MEDI & EP-DL \\
\hline 1 & RMSE & 75.0 & 40.5 & 39.5 \\
& HFEN & 61.6 & 33.4 & 34.3 \\
2 & RMSE & 64.6 & 37.1 & 34.3 \\
& HFEN & 63.2 & 30.6 & 30.5 \\
\hline
\end{tabular}

QSM, quantitative magnetic susceptibility mapping; EP-DL, edge prior guided dictionary learning; TKD, Thresholded K-space Division; MEDI, Morphology Enabled Dipole Inversion.

with $\chi_{R}$ and $\|\cdot\|_{F}$ denoting the reference and the Frobeniusnorm.

HFEN is defined by Eq. [9]:

$$
\sqrt{\left\|\operatorname{LoG}(\chi)-\operatorname{LoG}\left(\chi_{R}\right)\right\|_{F}^{2} /\left\|\operatorname{LoG}\left(\chi_{R}\right)\right\|_{F}^{2}}
$$

where LoG is the Laplacian of the Gaussian operator.

The RMSE and HFEN values of the reconstructed susceptibility maps are summarized in Tables 2,3 for the TKD, MEDI and EP-DL methods.

The results in Table 2 show that in the 2016 QSM reconstruction challenge data, the RMSE and HFEN of the 
Table 4 The RMSE and HFEN of two slices in Figure 3 by different methods for the 2016 QSM challenge data

\begin{tabular}{lccccc}
\hline \multirow{2}{*}{ Method } & \multicolumn{2}{c}{ MEDI } & & \multicolumn{2}{c}{ EP-DL } \\
\cline { 2 - 3 } \cline { 5 - 6 } & RMSE & HFEN & & RMSE & HFEN \\
\hline $80^{\text {th }}$ slice & 69.4 & 62.7 & & 56.4 & 55.4 \\
$85^{\text {th }}$ slice & 69.7 & 62.5 & & 57.2 & 53.1 \\
\hline
\end{tabular}

QSM, quantitative magnetic susceptibility mapping; EP-DL, edge prior guided dictionary learning; MEDI, Morphology Enabled Dipole Inversion.
Table 5 ROI error of MEDI and EP-DL methods for 2016 QSM challenge data reconstruction (ppm)

\begin{tabular}{llcccc}
\hline ROI & GP & PU & CN & RN & SN \\
\hline MEDI & 0.030 & 0.019 & 0.016 & 0.022 & 0.056 \\
EP-DL & 0.029 & 0.011 & 0.008 & 0.014 & 0.068 \\
\hline
\end{tabular}

QSM, quantitative magnetic susceptibility mapping; EP-DL, edge prior guided dictionary learning; MEDI, Morphology Enabled Dipole Inversion; GP, globus pallidus; PU. putamen; $\mathrm{CN}$, caudate nucleus; $\mathrm{RN}$, red nucleus; $\mathrm{SN}$, substantia nigra.
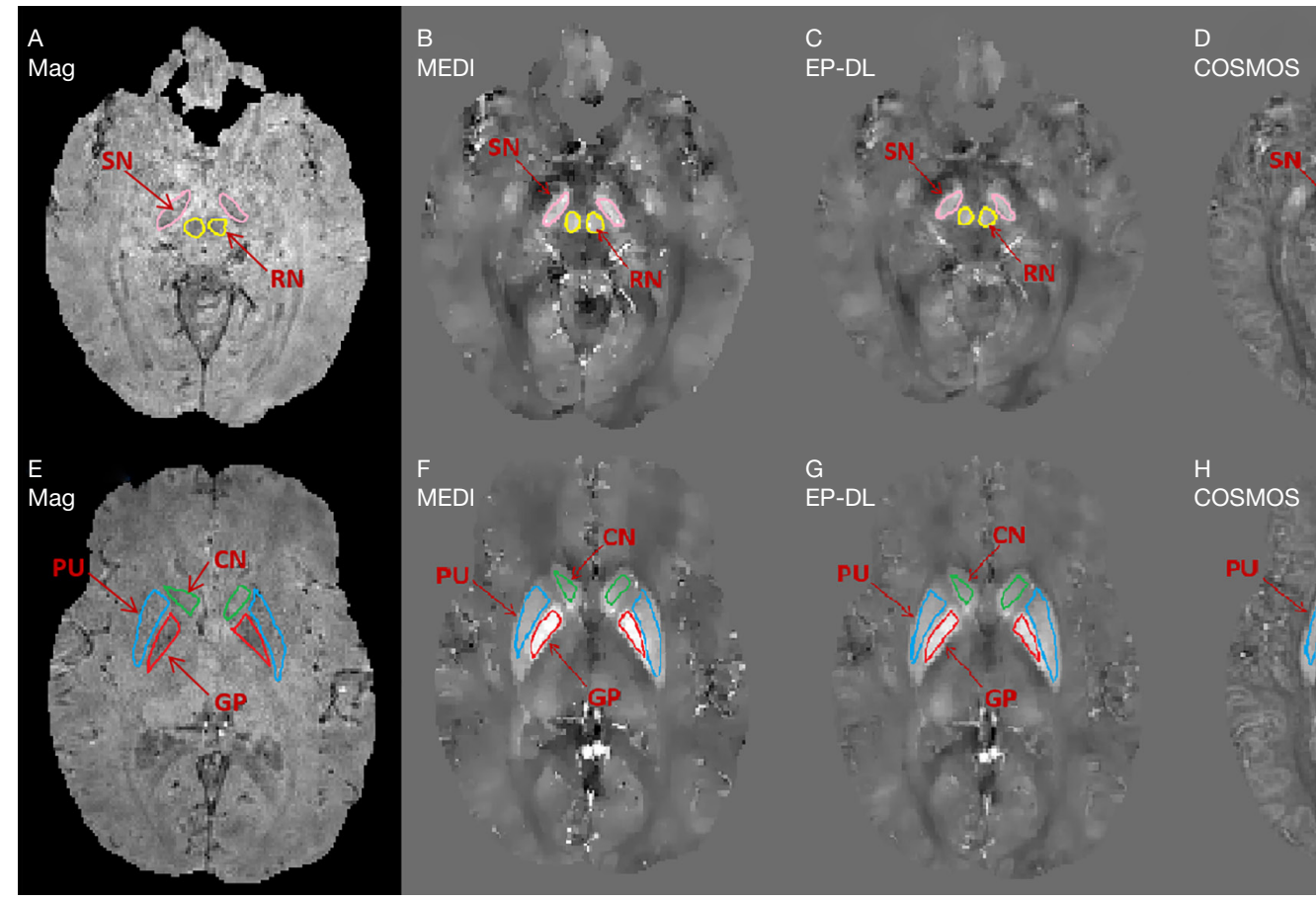

EP-DL

$\mathrm{H}$ cosmos

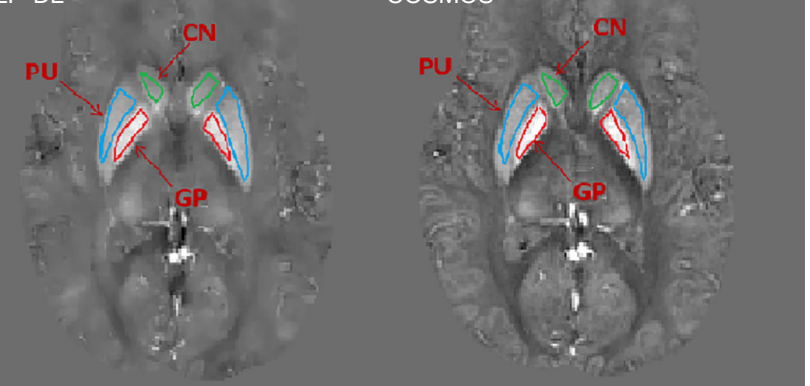

Figure 6 ROI of magnitude images and corresponding reconstructed susceptibility maps of MEDI (A,B), EP-DL (B,E) and COSMOS $(\mathrm{C}, \mathrm{F})$ method. Red is pallidus (GP), blue is putamen (PU), green is the caudate nucleus $(\mathrm{CN})$, yellow is the red nucleus $(\mathrm{RN})$ and pink is the substantia nigra (SN). The window/level is from -0.15 to $0.2 \mathrm{ppm}$.

EP-DL method are lower than the MEDI method, and the absolute improvements are $15.6 \%$ and $8.34 \%$ in terms of RMSE and HFEN, respectively.

Similarly, the EP-DL method also showed an advantage in the 2019 QSM reconstruction challenge data. For the first set, the quantitative indicators of EP-DL and MEDI were basically the same, and both were much lower than TKD. For the second set, the RMSE and HFEN of EP-DL were lower than those of TKD and MEDI.

In addition, Table 4 quantitatively evaluates the reconstruction performances of two slices showed in Figure 4. In the EP-DL method, the values of RMSE and HFEN are lower than the MEDI method. We also analyze the absolute values of the error in regions of interest (ROIs) for brain tissues of gray matter [globus pallidus (GP), putamen (PU), caudate nucleus $(\mathrm{CN})$, red nucleus $(\mathrm{RN})$, and substantia nigra $(\mathrm{SN})]$ relative to the reference COSMOS data.

ROI_error is defined as:

$$
R_{\text {RI_error }}=\operatorname{sum}\left(\operatorname{abs}\left(\chi_{R O I}-\chi_{R_{-} R O I}\right)\right) / R_{-} I_{-} p x
$$

where $\chi_{R O I}$ is the ROI of recovered susceptibility map, $\chi_{R \text { ROI }}$ is the ROI of reference and ROI_px is the pixel number of ROI. The range of the sum operation is the entire ROI.

Figure 6 depicts five selected ROI (Red is GP, blue is PU, 


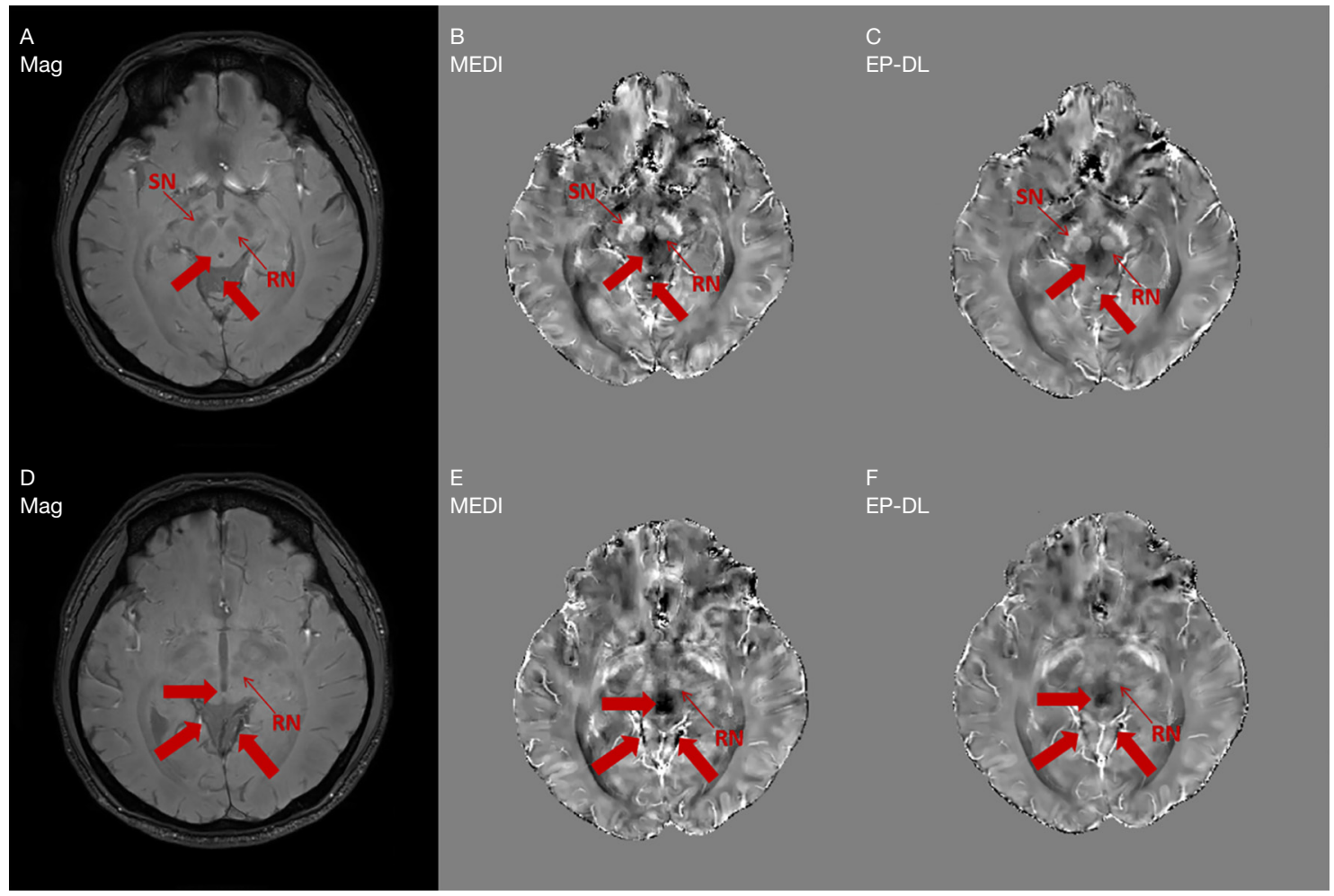

Figure 7 Magnitude images and corresponding QSM results of 3T Philips GRE data. Two selected slices of magnitude images (A,D) and the corresponding QSM reconstruction results from MEDI and EP-DL methods. We can observe that the streaking artifacts near ROI (RN, SN) in the MEDI method are better suppressed in the EP-DL method (where the red arrows indicate) than the MEDI method. The window/level is -0.15 to 0.15 ppm for QSM images. QSM, quantitative magnetic susceptibility mapping; EP-DL, edge prior guided dictionary learning; MEDI, Morphology Enabled Dipole Inversion; RN, red nucleus; SN, substantia nigra.

green is $\mathrm{CN}$, yellow is $\mathrm{RN}$ and pink is $\mathrm{SN}$ ) of reconstructed susceptibility maps by MEDI, EP-DL, and COSMOS method. Table 5 lists the absolute value of the error of these ROIs, and we can observe that in the EP-DL method, the ROI error of GP, PU, CN, and RN are lower than the MEDI method.

\section{Clinical GRE data results}

Additionally, two sets of single orientation in vivo brain data are used in algorithm evaluation. 3T GRE data is obtained from Philips MRI platform with acquisition parameters: FOV: $230 \mathrm{~mm} \times 230 \mathrm{~mm} \times 126 \mathrm{~mm}$, matrix size: $352 \times 352 \times 126, T R / T E_{1} / \Delta T E: 50 \mathrm{~ms} / 7.5 \mathrm{~ms} / 7.2 \mathrm{~ms}, \mathrm{FA}: 17^{\circ}$ and echo number: 6 . And $1.5 \mathrm{~T}$ GRE data is obtained from the XGY (an MRI equipment manufacturer in Ningbo, Zhejiang Province, China) MRI platform with acquisition parameters: FOV: $240 \mathrm{~mm} \times 192 \mathrm{~mm} \times 128 \mathrm{~mm}$, matrix size: $256 \times 205 \times 64, T R / T E_{1} / \Delta T E: 62 \mathrm{~ms} / 7.1 \mathrm{~ms} / 7.1 \mathrm{~ms}, \mathrm{FA}$ : $15^{\circ}$ and echo number: 4 .

The EP-DL and the MEDI methods are used to reconstruct the susceptibility maps of these two datasets. In the experiments, we find that in the EP-DL method, setting $\lambda_{2} / \lambda_{1}$, block size and dictionary atom number are the same as in Table 1 , and in the MEDI method, setting $\lambda_{2}$ to 1,000 can get good results visually. Improved performance in artifact suppression is also validated by the visual results in Figure 7 (3T Philips clinical data) and Figure 8 (1.5T XGY clinical data) for the EP-DL method, and the susceptibility mapping results use the same parameters given in Section III. A. This also confirms the robustness when the same parameter setting is used for the same data type in the proposed algorithm.

\section{Discussion}

In the proposed method, the parameters (regularization parameters $\lambda_{1}$ and $\lambda_{2}$, block size $\mathrm{N}$ and dictionary atom 


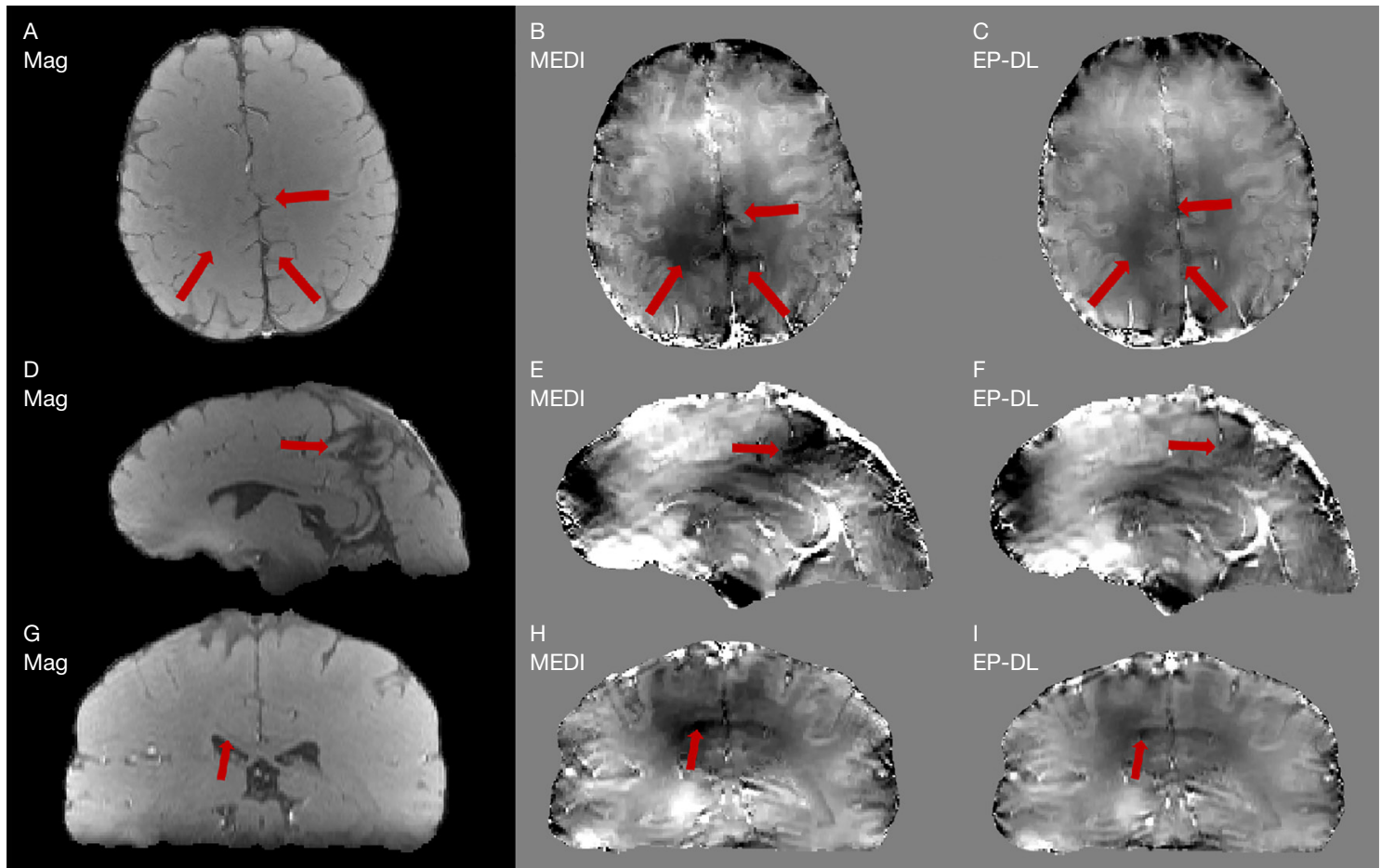

Figure 8 Magnitude images and corresponding QSM results of 1.5T XGY GRE data. The first, second, and last rows are the axial, sagittal and coronal, views, respectively. QSM reconstruction results by MEDI and EP-DL methods. MEDI method (B,E,H), EP-DL method $(\mathrm{C}, \mathrm{F}, \mathrm{I})$. From top to bottom are the axial, sagittal, and coronal views respectively. We can observe that streaking artifacts in the MEDI method are more effectively suppressed in the EP-DL method (the red arrows). The window/level is from -0.2 to 0.2 ppm for QSM images. QSM, quantitative magnetic susceptibility mapping; EP-DL, edge prior guided dictionary learning; MEDI, Morphology Enabled Dipole Inversion.

number $M_{2}$ ) are closely related to QSM reconstruction quality. Here the above parameters are analyzed with the other parameters fixed to the values given in Table 1 . Experiment setting. Experiment results also confirm that the same parameter setting can be used for the same type of dataset.

Eq. [5] has two regularization parameters $\lambda_{1}$ and $\lambda_{2}$, and the ratio between $\lambda_{1}$ and $\lambda_{2}$ needs to be well set to achieve a balance between different terms. With $\lambda_{1}$ ranging from 500 to 1,000, Figure 9 shows that the lowest RMSE and HFEN can be obtained when $\lambda_{2} / \lambda_{1}$ equals to 0.05 .

It can be observed in Figure 10 that the minimal RMSE and HFEN in the EP-DL method are obtained when $\lambda_{1}$ is set to 1,100 and 1,300, respectively. And the minimal RMSE and HFEN in the MEDI method are obtained when $\lambda_{1}$ equals 250 and 350 , respectively.

Nevertheless, in the experiment of 3T Philips data, when is set to 250, the MEDI method suffers from obvious oversmoothing (see in Figure 11). When varies from 600 to
1,400 , the obvious difference can be observed between these reconstructed susceptibility maps (see in Figure 11). Here, we denote as the difference map, and are reconstructed results with regularization parameters and, respectively. In the EP-DL method (is fixed to 0.05), when the value of is set from 800 to 2,000, the variations of quantitative metrics are found to be small for the 2016 QSM challenge data. And the lowest RMSE and HFEN are obtained when equals 1,100 and 1,300 (see in Figure 10A,10C), respectively. In the experiment of $3 \mathrm{~T}$ Philips data, in Figure 11, we can observe that in the EP-DL method, the reconstructed results are almost the same, and the difference between these susceptibility maps is smaller than the MEDI method.

Moreover, in the experiment of $1.5 \mathrm{~T} \mathrm{XGY} \mathrm{data,} \mathrm{in}$ Figure 12, we can observe that when varies from 600 to 1,400 , the difference between these reconstructed susceptibility maps by the MEDI method are obvious large than the EP-DL method (see the difference maps in Figure $12 D, 12 E, 12 I, 127)$. Compared with the MEDI method, the 


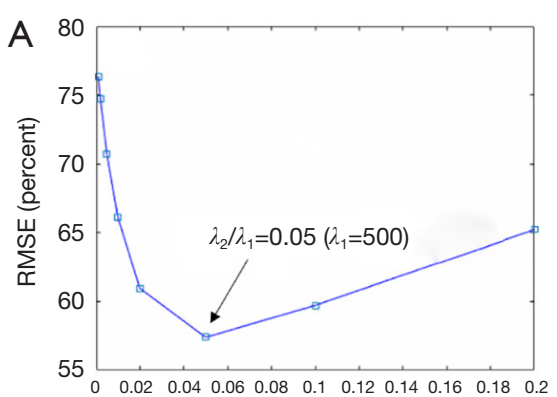

$\lambda_{2} / \lambda_{1}$

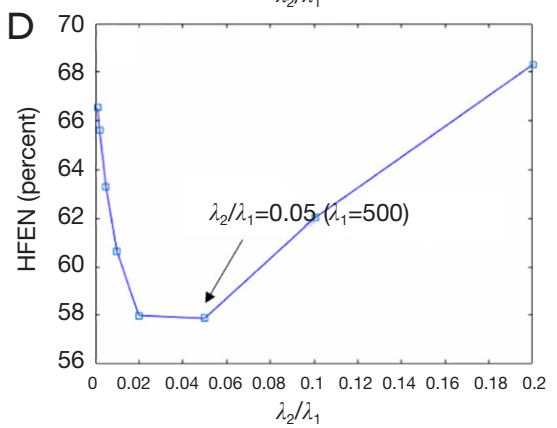

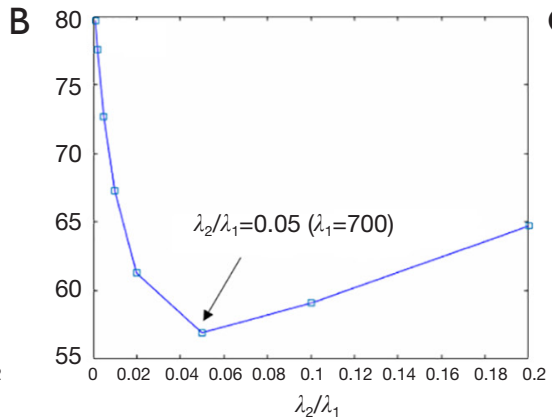

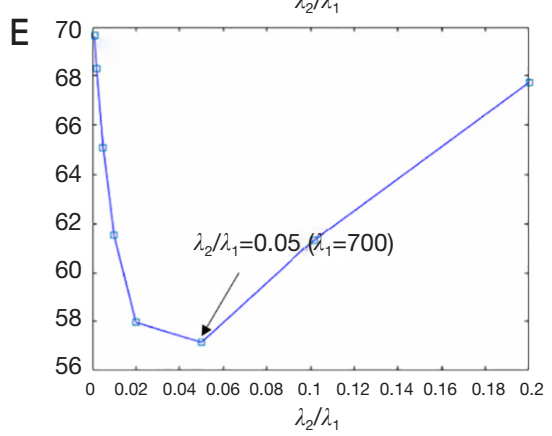

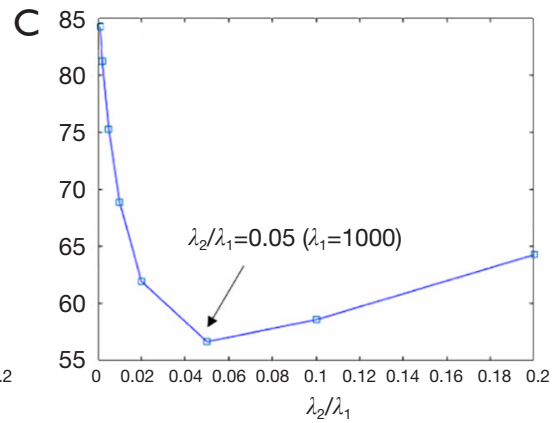

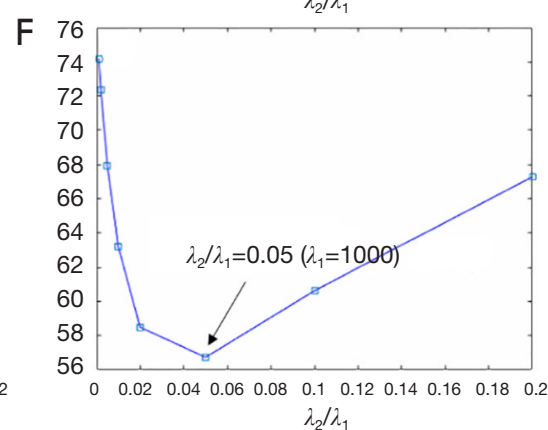

Figure 9 Analysis of the suitable ratio between regularization parameters $\lambda_{1}$ and $\lambda_{2}$ for EP-DL method, $\lambda_{1}$ set from 500 to 1,000 , from (A) to (D), we can observe that when $\lambda_{2} / \lambda_{1}$ equals 0.05 , RMSE and HFEN are always lowest.
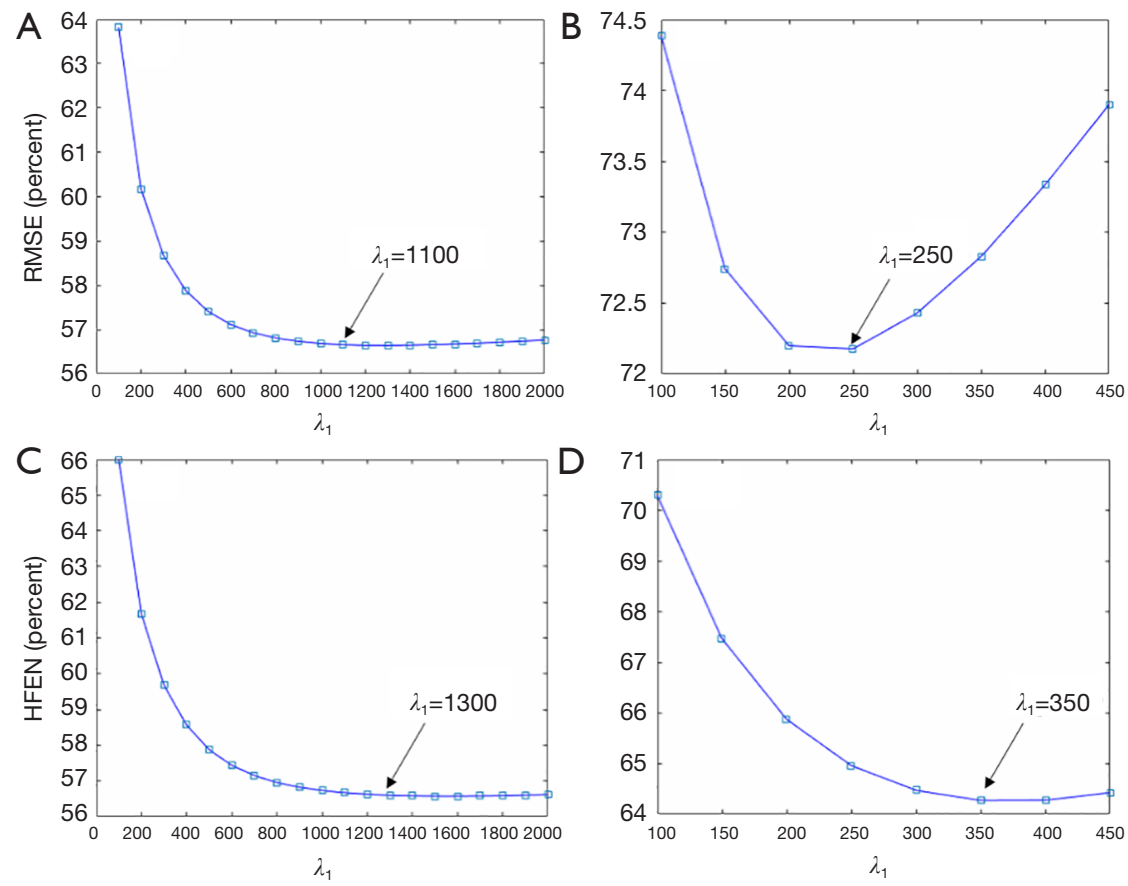

Figure 10 Analysis of regularization parameters $\lambda_{1}$ in EP-DL and MEDI method. The left column is the EP-DL method and $\lambda_{1}$ from 100 to 2,000, RMSE is minimum when $\lambda_{1}$ equals 1,100 and HFEN is minimum when $\lambda_{1}$ equals 1,300 . The right column is the MEDI method and $\lambda_{1}$ from 100 to 450 and RMSE is minimum when $\lambda_{1}$ equals 250 while HFEN is minimum when $\lambda_{1}$ equals 350 . EP-DL, edge prior guided dictionary learning; MEDI, Morphology Enabled Dipole Inversion. 


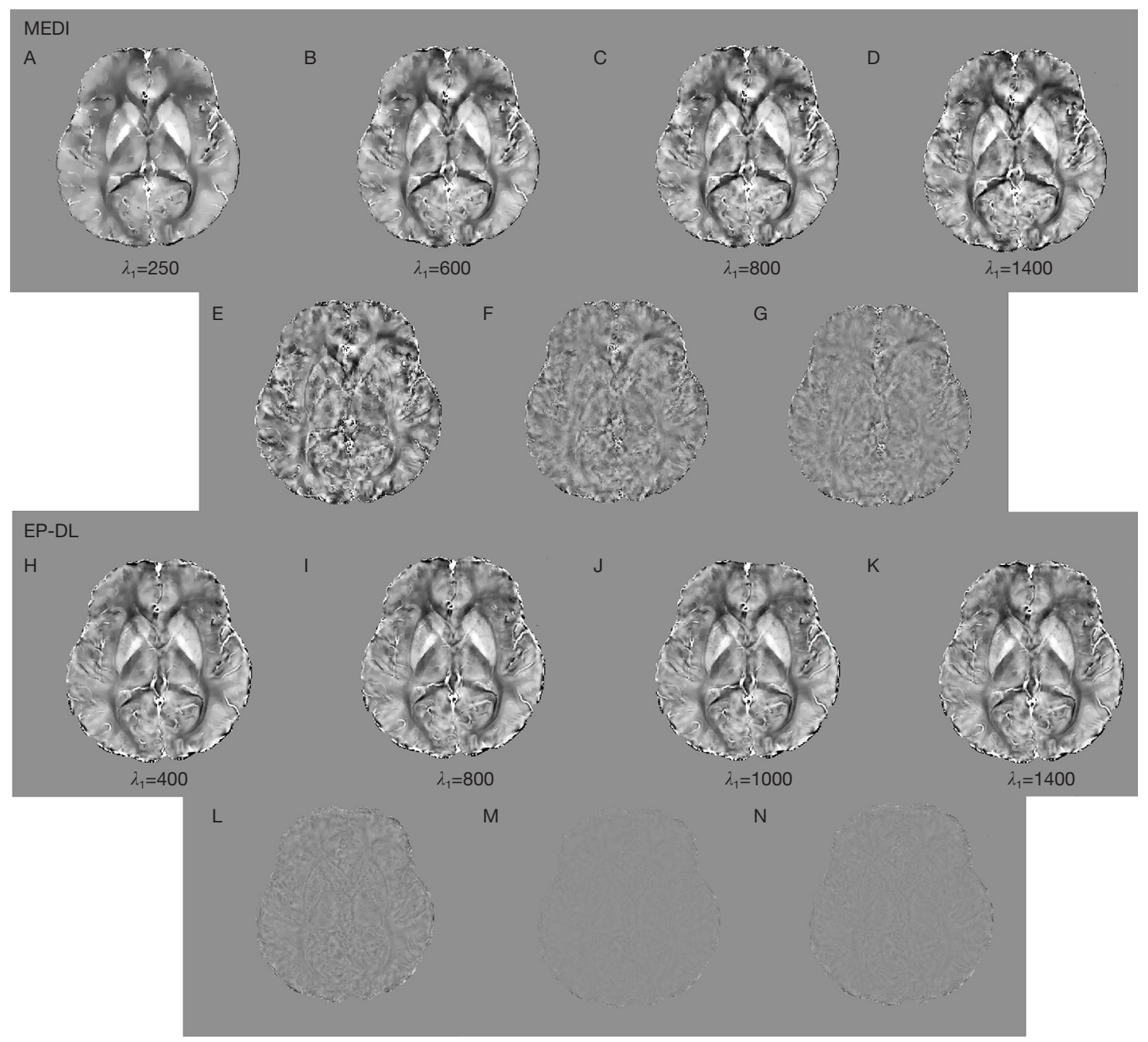

Figure 11 Reconstruction results for $3 \mathrm{~T}$ Philips data. (A) to (D) are the reconstructed results by the MEDI method, and regularization parameter $\lambda_{1}$ is set to 250, 600, 800 and 1,400, respectively, (E), (F) and (G) are the difference map between (A) and (B), (B) and (C), (C) and (D), respectively. $(\mathrm{H})$ to $(\mathrm{K})$ are reconstructed results by the EP-DL method, and regularization parameter $\lambda_{1}$ is set to 400 , 800 , 1,000 and 1,400, respectively, $(\mathrm{L}),(\mathrm{M})$ and $(\mathrm{N})$ are the difference maps between $(\mathrm{H})$ and $(\mathrm{I}),(\mathrm{I})$ and $(\mathrm{J}),(\mathrm{J})$ and $(\mathrm{K})$, respectively. The window/level is -0.15 to $0.15 \mathrm{ppm}$ for $(\mathrm{A})$ to $(\mathrm{D})$ and $(\mathrm{H})$ to $(\mathrm{K})$ while -0.05 to $0.05 \mathrm{ppm}$ for $(\mathrm{E})$ to $(\mathrm{G})$ and $(\mathrm{L})$ to $(\mathrm{N})$. EP-DL, edge prior guided dictionary learning; MEDI, Morphology Enabled Dipole Inversion.

proposed EP-DL method is found to be less sensitive to the regularization parameter when the parameter ratio is fixed.

The analysis of the block size $\mathrm{N}$ and dictionary atom number is provided in Figure 13 and Figure 14. Figure 13 shows that the minimal RMSE and HFEN can be obtained when the block size is set to $3 \times 3 \times 3$ and $5 \times 5 \times 5$, respectively. And we observe that the distance between the highest and lowest value of RMSE and HFEN are 0.69 and 1.39, respectively. In Figure 14, we can see that the minimum RMSE and HFEN are reached when the dictionary atom number $\mathrm{N}$ is set to 300 . We also find that the difference between the highest and lowest value of RMSE and HFEN are 0.23 and 0.19 , respectively. Such results show that the parameters $\mathrm{N}$ and have little effect upon the reconstruction quality in terms of metrics RMSE and HFEN.

For the 2016 QSM challenge data, EP-DL took about $10 \mathrm{~min}$ to train the dictionary using an Intel(R) Core(TM) i7-6820HK CPU. In terms of reconstruction times, EP-DL took about 10 minutes to reconstruct the QSM image, and the method of MEDI reconstructing time was about $5 \mathrm{~min}$. 


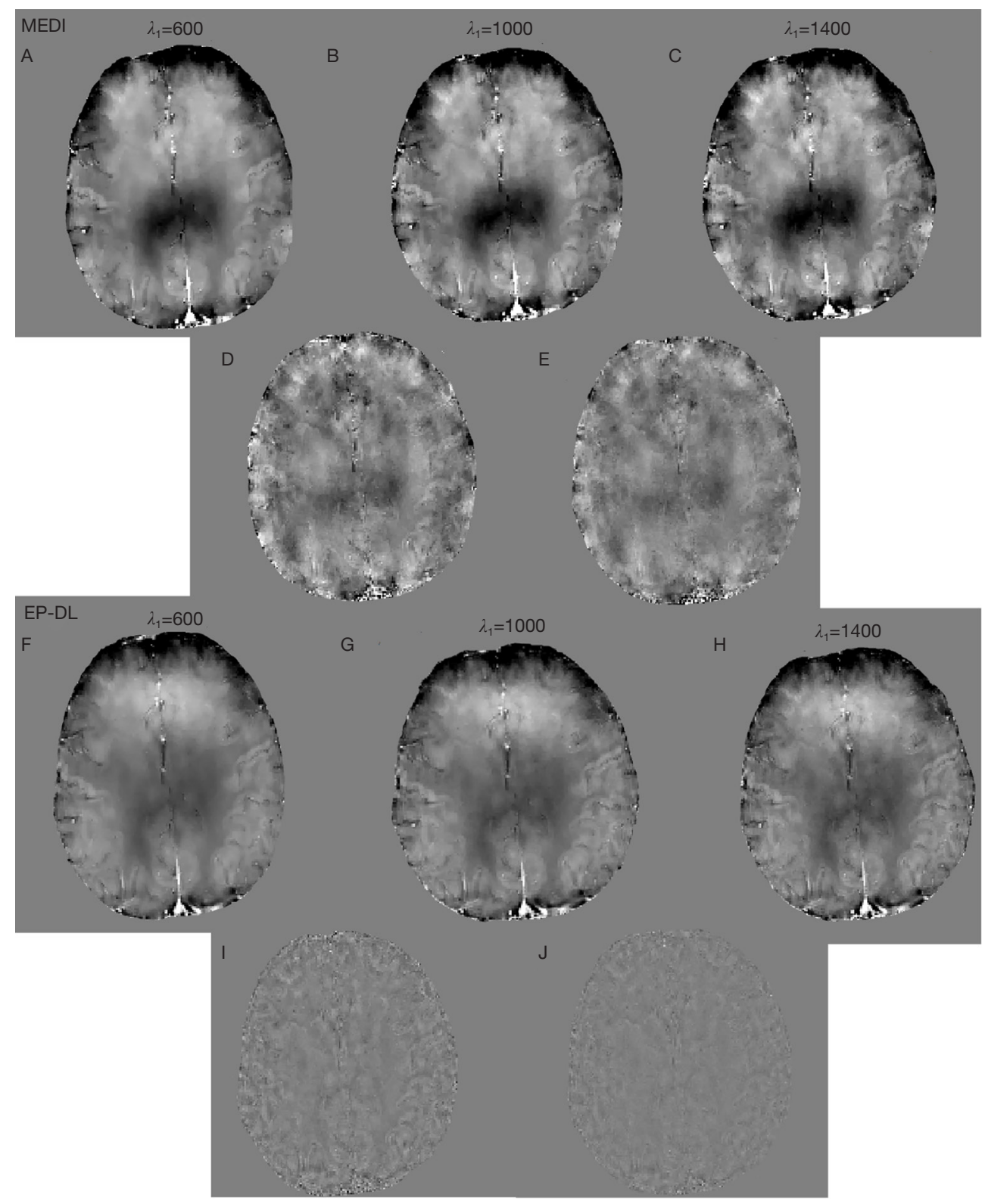

Figure 12 Reconstruction results of $1.5 \mathrm{~T}$ XGY data. (A) to (C) are the reconstructed results by the MEDI method, and the regularization parameter $\lambda_{1}$ is set to 600, 1,000, and 1,400, respectively, (D) and (E) are difference map between (A) and (B), (B) and (C), respectively. (F) to $(\mathrm{H})$ are reconstructed results by the EP-DL method, and the regularization parameter $\lambda_{1}$ is set to $600,1,000$, and 1,400, respectively, (I) and $(J)$ are the difference maps between $(F)$ and $(G),(G)$ and $(H)$, respectively. The window/level is -0.15 to $0.15 \mathrm{ppm}$ for $(A)$ to $(C)$ and $(F)$ to (H) while -0.05 to 0.05 ppm for (D), (E) and (I) and (J). EP-DL, edge prior guided dictionary learning; MEDI, Morphology Enabled Dipole Inversion.

\section{Conclusions}

In this paper, we propose an EP-DL method for dipole inversion in quantitative susceptibility mapping reconstruction. Improved performance in streaking artifacts suppression, structural recovery, deep gray matter reconstruction is obtained.

Future work will be focused on the evaluation of the effectiveness of the EP-DL method in patients with brain injuries such as micro-bleed multiple sclerosis 

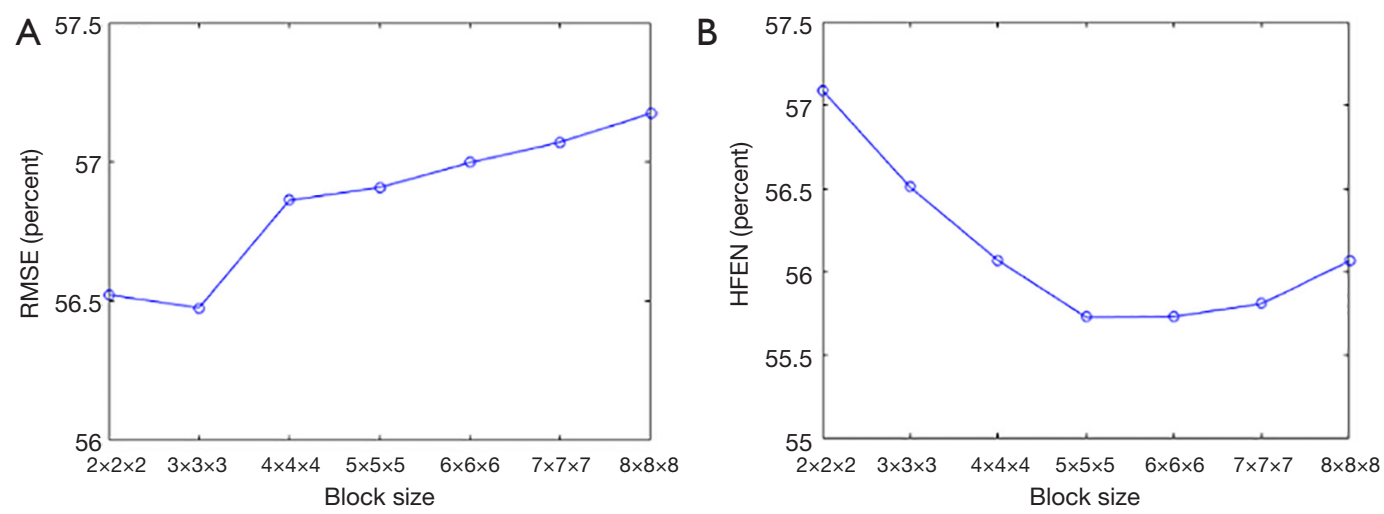

Figure 13 (A) is the RMSE curve of block size in EP-DL method for 2016 QSM challenge data, and correspondingly, (B) is the HFEN curve. Block size set from $2 \times 2 \times 2$ to $8 \times 8 \times 8$, we can observe RMSE is minimum when the block size set to $3 \times 3 \times 3$ and HFEN is minimum when the block set to $5 \times 5 \times 5$. QSM, quantitative magnetic susceptibility mapping; EP-DL, edge prior guided dictionary learning.
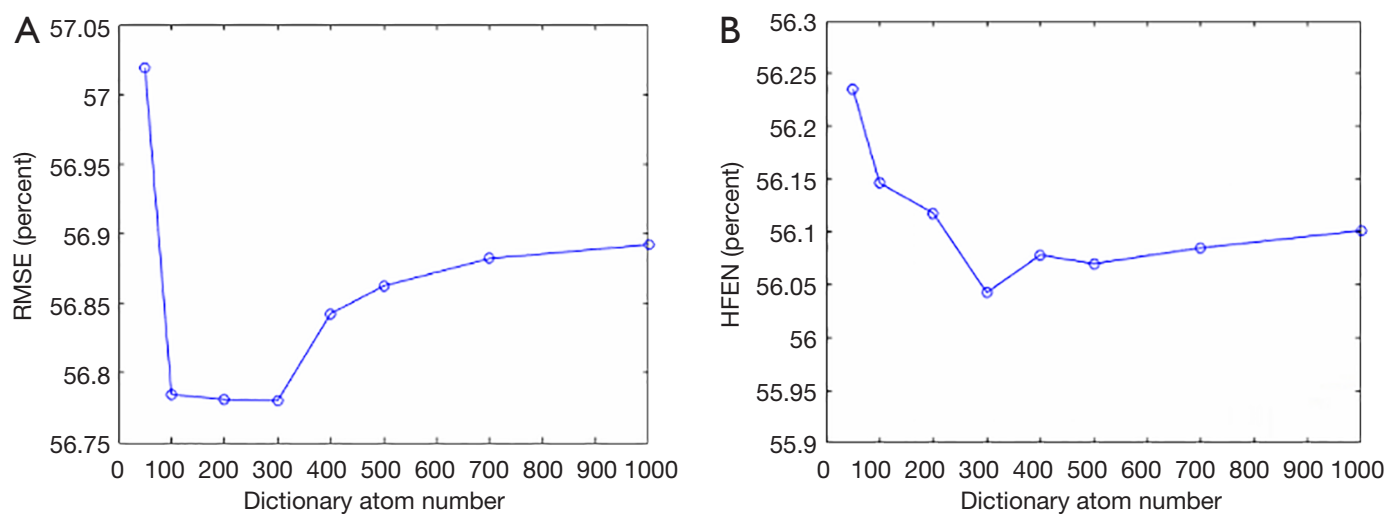

Figure 14 (A) is the RMSE curve of dictionary atom number in EP-DL method for 2016 QSM challenge data, and correspondingly, (B) is the HFEN curve. Dictionary atom number is set from 50 to 1,000, we can observe that both RMSE and HFEN change little. QSM, quantitative magnetic susceptibility mapping; EP-DL, edge prior guided dictionary learning.

lesions, hemorrhage, etc. To achieve a more robust QSM reconstruction, the adaptive parameters selection method needs to be developed for the data with different properties. Also, to meet the requirement of feasible applications, the iteration process needs to be accelerated by using parallelized computing architectures such as the Graphical Processing Unit (GPU).

\section{Acknowledgments}

Funding: This work was supported in part by the State's Key Project of Research and Development Plan under Grant 2017YFA0104302 and Grant 2017YFC0109202, in part by the National Natural Science Foundation under 61871117, in part by Science and Technology Program of Guangdong (2018B030333001).

\section{Footnote}

Conflicts of Interest: All authors have completed the ICMJE uniform disclosure form (available at https://dx.doi. org/10.21037/qims-21-243). The authors have no conflicts of interest to declare.

Ethical Statement: The authors are accountable for all aspects of the work in ensuring that questions related to the accuracy or integrity of any part of the work are appropriately investigated and resolved. The study was 
conducted in accordance with the Declaration of Helsinki (as revised in 2013). This study protocol was approved by the ethics committee of Southeast University, and written informed consent was obtained from each participant. All ethical guidelines were followed in our description of the experiments performed with human samples.

Open Access Statement: This is an Open Access article distributed in accordance with the Creative Commons Attribution-NonCommercial-NoDerivs 4.0 International License (CC BY-NC-ND 4.0), which permits the noncommercial replication and distribution of the article with the strict proviso that no changes or edits are made and the original work is properly cited (including links to both the formal publication through the relevant DOI and the license). See: https://creativecommons.org/licenses/by-nc-nd/4.0/.

\section{References}

1. Haacke EM, Tang J, Neelavalli J, Cheng YC. Susceptibility mapping as a means to visualize veins and quantify oxygen saturation. J Magn Reson Imaging 2010;32:663-76.

2. Wisnieff C, Ramanan S, Olesik J, Gauthier S, Wang Y, Pitt D. Quantitative susceptibility mapping (QSM) of white matter multiple sclerosis lesions: Interpreting positive susceptibility and the presence of iron. Magn Reson Med 2015;74:564-70.

3. Wang Y, Liu T. Quantitative susceptibility mapping (QSM): Decoding MRI data for a tissue magnetic biomarker. Magn Reson Med 2015;73:82-101.

4. Dimov AV, Liu Z, Spincemaille P, Prince MR, Du J, Wang Y. Bone quantitative susceptibility mapping using a chemical species-specific R2* signal model with ultrashort and conventional echo data. Magn Reson Med 2018;79:121-8.

5. Wong R, Chen X, Wang Y, Hu X, Jin MM. Visualizing and quantifying acute inflammation using ICAM-1 specific nanoparticles and MRI quantitative susceptibility mapping. Ann Biomed Eng 2012;40:1328-38.

6. Azuma M, Hirai T, Yamada K, Yamashita S, Ando Y, Tateishi M, Iryo Y, Yoneda T, Kitajima M, Wang Y, Yamashita Y. Lateral Asymmetry and Spatial Difference of Iron Deposition in the Substantia Nigra of Patients with Parkinson Disease Measured with Quantitative Susceptibility Mapping. AJNR Am J Neuroradiol 2016;37:782-8.

7. Liu T, Surapaneni K, Lou M, Cheng L, Spincemaille P, Wang Y. Cerebral microbleeds: burden assessment by using quantitative susceptibility mapping. Radiology 2012;262:269-78.

8. Sharma SD, Hernando D, Horng DE, Reeder SB. Quantitative susceptibility mapping in the abdomen as an imaging biomarker of hepatic iron overload. Magn Reson Med 2015;74:673-83.

9. Sun H, Klahr AC, Kate M, Gioia LC, Emery DJ, Butcher KS, Wilman AH. Quantitative Susceptibility Mapping for Following Intracranial Hemorrhage. Radiology 2018;288:830-9.

10. de Rochefort L, Liu T, Kressler B, Liu J, Spincemaille P, Lebon V, Wu J, Wang Y. Quantitative susceptibility map reconstruction from $M R$ phase data using bayesian regularization: validation and application to brain imaging. Magn Reson Med 2010;63:194-206.

11. Wharton S, Schäfer A, Bowtell R. Susceptibility mapping in the human brain using threshold-based k-space division. Magn Reson Med 2010;63:1292-304.

12. Li W, Wang N, Yu F, Han H, Cao W, Romero R, Tantiwongkosi B, Duong TQ, Liu C. A method for estimating and removing streaking artifacts in quantitative susceptibility mapping. Neuroimage 2015;108:111-22.

13. Schweser F, Sommer K, Deistung A, Reichenbach JR. Quantitative susceptibility mapping for investigating subtle susceptibility variations in the human brain. Neuroimage 2012;62:2083-100.

14. Fong D, Saunders M. LSMR: an iterative algorithm for sparse least-squares problems. SIAM J Sci Comput 2011;33:2950-71.

15. Langkammer C, Bredies K, Poser BA, Barth M, Reishofer G, Fan AP, Bilgic B, Fazekas F, Mainero C, Ropele S. Fast quantitative susceptibility mapping using 3D EPI and total generalized variation. Neuroimage 2015;111:622-30.

16. Wei H, Dibb R, Zhou Y, Sun Y, Xu J, Wang N, Liu C. Streaking artifact reduction for quantitative susceptibility mapping of sources with large dynamic range. NMR Biomed 2015;28:1294-303.

17. Liu Z, Kee Y, Zhou D, Wang Y, Spincemaille P. Preconditioned total field inversion (TFI) method for quantitative susceptibility mapping. Magn Reson Med 2017;78:303-15.

18. Sun H, Ma Y, MacDonald ME, Pike GB. Whole head quantitative susceptibility mapping using a leastnorm direct dipole inversion method. Neuroimage 2018;179:166-75.

19. Liu T, Liu J, de Rochefort L, Spincemaille P, Khalidov I, Ledoux JR, Wang Y. Morphology enabled dipole inversion (MEDI) from a single-angle acquisition: comparison with 
COSMOS in human brain imaging. Magn Reson Med 2011;66:777-83

20. Liu T, Wisnieff C, Lou M, Chen W, Spincemaille P, Wang Y. Nonlinear formulation of the magnetic field to source relationship for robust quantitative susceptibility mapping. Magn Reson Med 2013;69:467-76.

21. Liu T, Spincemaille P, de Rochefort L, Kressler B, Wang Y. Calculation of susceptibility through multiple orientation sampling (COSMOS): a method for conditioning the inverse problem from measured magnetic field map to susceptibility source image in MRI. Magn Reson Med 2009;61:196-204.

22. Yoon J, Gong E, Chatnuntawech I, Bilgic B, Lee J, Jung W, Ko J, Jung H, Setsompop K, Zaharchuk G, Kim EY, Pauly J, Lee J. Quantitative susceptibility mapping using deep neural network: QSMnet. Neuroimage 2018;179:199-206.

23. Bollmann S, Rasmussen KGB, Kristensen M, Blendal RG, Østergaard LR, Plocharski M, O'Brien K, Langkammer C, Janke A, Barth M. DeepQSM - using deep learning to solve the dipole inversion for quantitative susceptibility mapping. Neuroimage 2019;195:373-83.

24. Liu J, Chen Y, Hu Y, Luo L. Low-dose CBCT reconstruction via 3D dictionary learning. 2016 IEEE 13 th International Symposium on Biomedical Imaging (ISBI), Prague, pp. 735-738, Apr. 2016.

25. Chen Y, Shi L, Feng Q, Yang J, Shu H, Luo L, Coatrieux JL, Chen W. Artifact suppressed dictionary learning for low-dose CT image processing. IEEE Trans Med Imaging 2014;33:2271-92.

26. Liu J, Hu Y, Yang J, Chen Y, Shu H, Luo L, Feng Q, Gui Z, Coatrieux G. 3D Feature Constrained Reconstruction for Low Dose CT Imaging. IEEE Transactions on Circuits and Systems for Video Technology 2018;28:1232-47.

27. Liu J, Ma J, Zhang Y, Chen Y, Yang J, Shu H, Luo L,
Coatrieux G, Yang W, Feng Q, Chen W. Discriminative Feature Representation to Improve Projection Data Inconsistency for Low Dose CT Imaging. IEEE Trans Med Imaging 2017;36:2499-509.

28. Ravishankar S, Bresler Y. MR image reconstruction from highly undersampled k-space data by dictionary learning. IEEE Trans Med Imaging 2011;30:1028-41.

29. Zhuang P, Zhu X, Ding X. MRI reconstruction with an edge-preserving filtering prior. Signal Processing, 2019;155:346-57.

30. Iyer SK, Tasdizen T, Dibella E. Edge-enhanced spatiotemporal constrained reconstruction of undersampled dynamic contrast-enhanced radial MRI. Magn Reson Imaging 2012;30:610-9.

31. Langkammer C, Schweser F, Shmueli K, Kames C, Li X, Guo L, Milovic C, Kim J, Wei H, Bredies K, Buch S, Guo Y, Liu Z, Meineke J, Rauscher A, Marques JP, Bilgic B. Quantitative susceptibility mapping: Report from the 2016 reconstruction challenge. Magn Reson Med 2018;79:1661-73.

32. Elad M, Aharon M. Image denoising via sparse and redundant representations over learned dictionaries. IEEE Trans Image Process 2006;15:3736-45.

33. Rubinstein R, Zibulevsky M, Elad M. Efficient implementation of the K-SVD algorithm using batch orthogonal matching pursuit. CS Technion 2008;40:1-15.

34. Jenkinson M, Beckmann CF, Behrens TE, Woolrich MW, Smith SM. FSL. Neuroimage 2012;62:782-90.

35. Schofield MA, Zhu Y. Fast phase unwrapping algorithm for interferometric applications. Opt Lett 2003;28:1194-6.

36. Zhou D, Liu T, Spincemaille P, Wang Y. Background field removal by solving the Laplacian boundary value problem. NMR Biomed 2014;27:312-9.
Cite this article as: Du J, Ji Y, Zhu J, Mai X, Zou J, Chen Y, $\mathrm{Gu}$ N. Edge prior guided dictionary learning for quantitative susceptibility mapping reconstruction. Quant Imaging Med Surg 2022;12(1):510-525. doi: 10.21037/qims-21-243 